\title{
Effect of Some Fruits as feed Additives and their role Against Hyperlipidemia and Obesity of Experimental rats
}

\section{Khadega Mohamed Sayed}

$(*)$

\section{SUMMARY}

Hyperlipidemia and obesity are associated with many diseases which badly affective on health. Increased consumption of fruits is widely recommended to improve health and decrease overweight and the level of lipid in the blood.

The aim of the present work is to study the effect of diet containing (apple, kiwi and combination of them) on the nutritional evaluation and biochemical parameters of rats. Suffering from high level of lipid and obesity. Seventy two albino male rats weighing $119 \pm 2 \mathrm{~g}$ used in this study. All rats fed on basal diet for two week for adaptation, after this period, rats divided in to two main groups, the first main group (8rats) fed on basal diet throughout the experimental periods (4 months) and considered as a negative control group, while the second main group fed on high fat diet for two months, then this group( $n=64$ rats) was divided in to eight subgroups ( $\mathrm{n}=8$ rats of each ). The first four subgroups were continued fed on high fat diet with apple, kiwi and combination of them. However the other four subgroups fed on basal diet with apple, kiwi and combination of them. The eight subgroups were as followed, subgroup (1) positives control $(1)$ fed on high fat diet .subgroups $(2,3,4)$ fed on high fat diet containing (apple, kiwi and combination of them) respectively, subgroup(5) positive control (2) fed on basal

$\left({ }^{*}\right)$ M.Sc, Nutrition and Food Science, Faculty of Specific Education, Minia University. 
diet. Subgroups $(6,7,8)$ fed on basal diet containing (apple, kiwi and combination of them) respectively.

The results revealed that found a significant increase in the body weight from positive control of high fat diet rat and obesity rat groups. Also increase a significant the level of lipid profile, liver enzymes and kidney function compared to negative control grope. While when feeding rats with high fat diet containing apple, kiwi and combination of them occur significant decrease in body weigh, liver enzymes, kidney function and level of lipid profile ( TC, TG, TL and LDL) and increase the level of HDL. And also when feeding obese rats with basal diet containing apple, kiwi and combination of them lead to significant decrease and improve body weigh, kidney function, liver enzymes and lipid profile (TC, TG, TL, LDL) and increase the level of HDL. In Histopathological study we find out change in cells liver, kidney, heart and brain in rats.

\section{Key word: - Hyrerlipidemia, obesity, apple and}

\section{kiwi.}

Hyperlipidemia is one of the main causes of half of deaths from cardiovascular disease. Lipids leading to obesity which it is a medical condition in which excess body fat or white adipose tissue and accumulates in the body to the extent that this accumulation of fat might adversely affect health, potentially.

\section{Therefore, the study aimed to:}

- Evaluate the effect of some fruits to experimental rats diets on the level of lipids profile in the blood. 
- Study the effect of some fruits to experimental rats diets on obesity.

- Attempt to use the fruits in making a new biscuits.

\section{Sample study:}

1) Apple and kiwi were purchased from a local market, Cairo, Egypt in March and April 2014. The clean parts were dried at $40-45 \mathrm{C}$ in oven under vacuum.

2) Animal fat were obtained from a local market, Minia, Egypt. They cut, heated and filtered several times.

\section{Animals and experimental design:}

Seventy two albino male rats weighing $119 \pm 2 \mathrm{~g}$ were obtained from Agricultural faculty, Minia Univ. Egypt. And were acclaimed for two week prior to experiment. They were housed in special healthy plastic cages and maintained on $a d$ libitum for water and free access of basal diet, at room temperature. All groups with out the negative control group were fed on high fat diet for the first two months and after then they were fed on the next:

Group ( 1 ) : Negative control (C -); rats fed on basal diet.

Group ( 2 ) : Positive control $(\mathrm{C}+1)$; were fed on high fat diet composec diet $+10 \%$ animal fat.

Group ( 3 ) : Rats fed on high fat diet $+20 \%$ dried apple (AP1).

Group ( 4 ) : Rats fed on high fat diet $+20 \%$ dried kiwi (Ki1).

Group ( 5 ) : Rats fed on high fat diet $+20 \%$ combination of dried (apple 
(AK1).

Group ( 6 ) : Positive controls $(\mathrm{C}+2)$ were fed on basal diet only after the months.

Group ( 7 ) : Rat fed on basal diet $+20 \%$ dried apple (AP2).

Group ( 8 ) : Rat fed on basal diet $+20 \%$ dried kiwi (KI2).

Group (9): Rat fed on basal diet $+20 \%$ combination of (apple a (AK2).

At the end of the experiment, all animal were fasted for $12 \mathrm{~h}$ and then blood sample were collected under diethyl 1 ether. Each sample was centrifuged at $3000 \mathrm{rpm}$ for 15 Min, the obtained supernatant (serum) kept at $-20 \mathrm{C}$ until used in biochemical analysis. Lipid profile cholesterol, triglycerides ( TG ), high density lipoprotein ( HDL-C ), low density lipoprotein ( $\mathrm{LDL}-\mathrm{C}$ ), total lipid ( TL ), lipid peroxide, glutamic oxaloacetic transaminas ( GOT ), glutamic pyruvic transamines ( GPT), alkaline phosphates ( ALP ), Nitric oxide, urea, protein and albumin.

At the same time, the organs : ( Liver, Kidney, heart and brain ) were removed, washed in saline solution, dried by filter paper, weighted and stored frozen in formalin solution $10 \%$ for histopathological examinations.

- Statistical analysis were performed by SPSS program.

Results can be summarized biological experiment as follows: 
Effect of Some Fruits as feed Additives and their role Against Hyperlipidemia and Obesity of Experimental rats

(A)- The results for high fat diet rat can be summarized

\section{biological experiment as follows:-}

\section{1) The average body weight gain and weight of organs:}

The result of the experiment showed that the positive control $(\mathrm{C}+1)$ groups of rats which fed on high fat diet increase in body weight and member of mice compared to negative control (C -). And groups fed on high fat diet supplemented with apple, kiwi (KI) and combination of them there was decrease in body weight of mice and member compared to positive control group.

2) Some biochemical parameters in blood serum of rat fed on high fat diet apple, kiwi and combination of them:-

- Total lipid, lipid peroxide, cholesterol (beneficial and harmful) triglycerides in the blood serum of rats.

The results showed that rats in positive control group which fed on high fat diet lead to a significant increase in the level of triglyceride, total lipid, lipid peroxide and total cholesterol compared to negative control group. The results also showed a significant decrease in the level of triglyceride, total lipid, lipid peroxide and total cholesterol in groups that fed on high fat diet containing apple, Kiwi and combination of them compared to positive control group.

The results also showed a significant decrease in the level of high density lipoprotein (HDL) in the positive 
control group compare to negative control group. While the groups which fed on high fat diet containing apple, kiwi and combination of them showed significant increase in the level of high density lipoprotein (HDL) compared to positive control group.

\section{- Liver enzymes functions:}

The results showed that rats in positive control which fed on high fat diet lead to a significant increase in the level of (GOT, GPT) compared with negative control group. While observed when rats fed on high fat diet containing (apple, kiwi and combination of them) showed significant decrease in the level of (GOT - GPT) compared to positive control group.

\section{3- Histopathological changes:}

\section{Liver:}

The results of microscopic examination of the liver that rats of positive control which fed on high fat diet showed fatty change of hepatocytes and sinusoidal leucocytosis. While, the best result of liver in groups which fed on high fat diet contained (apple, kiwi and combination of them) in group which fed on high fat diet contained kiwi and combination of apple and kiwi respectively showed no histophathological changes.

\section{Kidney:}


Histopathologicaly, kidney of positive control showed congestion of intertubular blood vessels and vacuolation of endothelial lining glomerular tuft. While, the best result of kidney in groups which fed on high fat diet contained (apple, kiwi and combination of them) in group which fed on high fat diet contained kiwi showed no histophathological changes.

\section{Heart:}

Histopathologically heart of rat from positive control group showed intermyocardial oedema. However heart of rat groups which fed on high fat diet contained (apple, kiwi and combination of them) showed no histopathological changes.

\section{Brain:}

Brain of the positive control showed congestion of blood vessel. While, the best result of brain in groups which fed on high fat diet contained (apple, kiwi and combination of them) in group which fed on high fat diet contained kiwi and combination of apple and kiwi respectively showed no histophathological changes.

\section{(B) The results for low fat diet rat can be summarized}

\section{biological experiment as follows:-}

\section{1 - The average body weight gain and weight of organs:-}

The results of the experiment showed that positive control groups of rats which fed on basal diet increase in body weight and member of mice compared to negative 
control. And group fed on basal diet supplemented with apple, kiwi and combination of them there was decrease in body weight of mice and member compared to positive control group.

$\underline{2}$ - Some biochemical parameters in blood serum of rat fed on basal diet contained apple, kiwi and combination of them:-

- Total lipid, lipid peroxide, cholesterol (beneficial and harmful), triglycerides in the blood serum of rats:-

The results showed that rats in positive control group which fed on basal diet lead to a significant increase in the level of triglyceride, total lipid, lipid peroxide and total cholesterol compared to negative control group. The results also showed a significant decrease in the level of triglycerides, total lipid, lipid peroxide and total cholesterol in groups that fed on basal diet containing apple, kiwi and combination of them compared to positive control group.

The results also showed that positive control group fed on basal diet lead to a significant increase in the level of low density lipoprotein (LDL) compared to negative control group. While it was noted that the group fed on basal diet containing apple, kiwi and combination of them showed 
significant decrease in the level of low density lipoprotein (LDL) compared to positive control group.

The results also showed a significant decrease in the level of high density lipoprotein (HDL) in the positive group compared to negative control group. While it groups which fed on basal diet containing apple, kiwi and combination of them showed significant increase in the level of high density lipoprotein (HDL) compared to positive control group.

\section{- Liver enzymes functions:}

The results showed that rats in positive control group which fed on basal diet lead to a significant increase in the level of (GOT, GPT) compared with negative control group. While observed when fed rats on basal diet containing apple, kiwi and combination of them significant decrease in (GOT, GPT) compared to positive control group.

\section{3-Histopathological changes:}

\section{Liver:}

The results of microscopic examination of the liver that rats of positive control showed kupffer cell activation. While, the best result of liver in groups which fed on basal diet contained (apple, kiwi and combination of them) in group which fed on basal diet contained kiwi showed no histophathological changes.

\section{Kidney:}


The results of microscopic examination of the kidney of positive control showed slight congestion of glomerular tufts. While, the best result of kidney in groups which fed on basal diet contained (apple, kiwi and combination of them) in groups which fed on basal diet contained apple and kiwi respectively showed no histophathological changes.

\section{Heart:}

Histopathologically heart of rat from positive control group showed congestion of myocardial blood vessel. While, the best result of heart in groups which fed on basal diet contained (apple, kiwi and combination of them) in group which fed on basal diet contained apple and group which fed on basal diet contained kiwi showed no histophathological changes.

Sensory evaluation of biscuit produced from wheat flour, starch and dried

\section{(apple, kiwi and combination of them).}

The study showed that the best results for colour, odour and texture in the biscuit which supplemented with kiwi and starch followed by biscuit which supplemented with combination of apple and kiwi and starch. And the lowest results for biscuit which supplemented with apple. While the biscuit which supplemented with combination of apple and kiwi had the best result of appearance and acceptance. 


\section{The study concluded the following recommendations:-}

1) We have to consume fruit (apple and Kiwi) regularly in our daily because they contain fiber which can lower the risk of coronary heart disease.

2) Eating Apple and Kiwi to improve HDL - C in the blood.

3) Nutrition programs about using fruits in decreasing the risk of obesity and hyperlipedimia.

4) Support biscuits with fruit (apple and Kiwi) to avoid rising of lipid and obesity between diets (Meals). 